\title{
ALGUNOS PROBLEMAS DEL ANÁLISIS DE FORMACIONES INTERFIJADAS Y PARASINTÉTICAS EN ESPAÑOL
}

\author{
Petr Stehlik
}

\begin{abstract}
This article examines three aspects closely related to the mechanisms of interfixation and parasynthesis in Spanish: 1) the importance of diachronic approach for analyzing parasynthetic and interfixed formations; 2) the semantic value of the affix in question; and 3) the consequences of the interpretation of the final segment as derivative or inflectional morpheme in parasynthetic and interfixed verbs. According to our research, the concepts of interfixation and parasynthesis are both based on a diachronic perspective, which makes difficult the application of purely synchronous methods to analyze words formed by these processes. Another problem in common is that in both procedures, it is difficult to determine the specific semantic value of the affix (interfix or prefix). As for the third above-mentioned aspect, the interpretation of the verbal suffix (especially -ar) as inflectional morpheme necessarily leads to the redefinition of all parasynthetic and interfixed verbs, turning these into suffixed or prefixed words, respectively.

Keywords: linguistics; morphology; Spanish; interfixation; parasynthesis.

Resumen: En este artículo se examinan tres aspectos relacionados con los mecanismos de interfijación y parasíntesis en español: 1) la importancia del enfoque diacrónico para el análisis de formaciones interfijadas y parasintéticas; 2) el valor semántico del formante en cuestión; y 3) las consecuencias de la interpretación del segmento final como morfema derivativo o flexivo en verbos supuestamente interfijados y parasintéticos. Según nuestra investigación, tanto el concepto de interfijación como el de parasíntesis están definidos desde una perspectiva diacrónica, lo que dificulta la aplicación de criterios puramente sincrónicos en el análisis de palabras formadas mediante estos mecanismos. Otro problema en común es que en ambos procedimientos resulta difícil determinar el valor semántico concreto del afijo (interfijo o prefijo). En cuanto al tercer aspecto mencionado, la interpretación del sufijo verbal (sobre todo -ar) como morfema flexivo llevaría necesariamente a la redefinición de todos los verbos interfijados y parasintéticos y a su consiguiente análisis como voces sufijadas o prefijadas, respectivamente.
\end{abstract}

Palabras claves: lingüística; morfología; español; interfijación; parasíntesis.

\section{Introducción}

Aunque los límites que separan los principales procedimientos de formación de palabras (prefijación, sufijación y composición) no son nítidos y parecen existir zonas de transición entre prácticamente todos ellos, hay dos mecanismos en especial -la interfijación y la parasíntesis- cuya propia existencia ha sido cuestionada y que difieren de los procesos antes mencionados por algunas propiedades de gran interés teórico. 
El concepto de parasíntesis cuenta, al menos en la lingüística románica, con una tradición mucho más larga que el de interfijación ${ }^{1}$, pero como veremos más adelante, detrás de algunas dificultades en el análisis morfológico de determinadas formaciones supuestamente interfijadas o parasintéticas subyacen causas muy similares, si no idénticas. Fue precisamente este denominador común el que nos llevó a examinar conjuntamente los dos procesos y centrarnos en los siguientes puntos problemáticos:

1) la necesidad de recurrir a la perspectiva diacrónica para determinar el estatuto de las palabras real o aparentemente parasintéticas e interfijadas;

2) la aportación semántica del afijo en las formaciones en cuestión;

3) el carácter flexivo o derivativo del morfema final en los verbos parasintéticos e interfijados (p. ej. engordar, apretujar).

\section{Definiciones}

Antes de entrar en la materia, consideramos útil esbozar primero, al menos brevemente, los conceptos de parasíntesis e interfijación.

La parasintesis consiste, según la NGLE (2009: 577-578), «en la adición de AFIJOS DISCONTINUOS, en concreto un prefijo y un sufijo elegidos simultáneamente». Ya el término afijo discontinuo, empleado en la última gramática académica, es polémico, pues según destacados morfólogos españoles (Serrano-Dolader 1995, 1999; Varela 2005) no se trata, en este proceso, de la adjunción de un solo afijo discontinuo (llamado también circunfijo) sino de dos morfemas independientes: un prefijo y un sufijo, eso sí, unidos simultáneamente a la base (p. ej. pobre>em-pobr-ecer, luna>a-lun-izar). Dado que en los trabajos publicados en el último decenio se suele restringir el alcance del término parasíntesis a la aplicación simultánea de dos afijos derivativos a la base (Pref+B+Suf), no nos ocupamos aquí de la parasíntesis por composición (ropa vieja>ropavejero, siete meses>sietemesino, etc.), mucho menos productiva que el primer tipo mencionado.

En cuanto a la interfijación, esta se define como la inserción de un morfema (interfijo) entre la base y el sufijo. Según ya hemos señalado, el concepto de interfijación fue planteado con cierto retraso respecto al estudio de las formaciones parasintéticas y, lo que es más, fue propuesto específicamente para el español ${ }^{2}$ aunque pueden encontrarse elementos lingüísticos con las mismas características y funciones también en otras lenguas románicas (el francés, el portugués, el italiano ${ }^{3}$ ).

$\mathrm{Al}$ igual que en la parasíntesis, en la interfijación es crucial la inexistencia de la fase intermedia de la formación resultante, pues cuando se adjunta un sufijo a una palabra previamente sufijada, estamos ante una mera cadena sufijal en vez de ante una combinación de un interfijo y sufijo.

Curiosamente, parece que el papel de la interfijación en la morfología española va relativizándose en los últimos años: mientras que en la GDLE (1999) se dedica a este mecanismo todavía un extenso estudio ${ }^{4}$, en la NGLE (2009) se hace mención de la interfijación

1 El francés A. Darmesteter (1875) se ocupó de la parasíntesis ya en la segunda mitad del siglo XIX, mientras que los primeros trabajos sobre la interfijación aparecen tan solo en los años 50 del siglo pasado (Malkiel 1958).

2 Significativamente, Malkiel (1958) tituló su más importante artículo sobre la interfijación «Los interfijos hispánicos. Problema de lingüística histórica y estructural».

3 Štichauer (2007: 69) menciona varias palabras italianas que incluyen un interfijo, p. ej. gita>gitarella, mangione>mangioncello.

4 Al contrario, la parasíntesis se menciona en la GDLE (1999) en varios capítulos sin reservársele un espacio aparte, tal como se solía hacer en las gramáticas y tratados sobre la formación de palabras anteriores. 
solo de paso en los capítulos sobre la derivación nominal, adjetiva y apreciativa. También la postura de los académicos hacia los interfijos ha cambiado sustancialmente, como se aprecia en el siguiente comentario acerca de la derivación adjetival en la que intervienen interfijos (NGLE 2009: 511): «Se observa en café>cafetero que se añade el segmento -t-, entre la base y el sufijo. Estos segmentos, a los que no corresponde propiamente un significado, se suelen denominar INTERFIJOs en la tradición morfológica. No existe acuerdo entre los gramáticos acerca de si son unidades morfológicas independientes (cafe-t-ero), han de considerarse parte de la base alternante (cafet-ero) o bien parte del afijo (cafe-tero). Se suele aceptar hoy que las dos primeras opciones son más adecuadas que la tercera». Esta opinión considerablemente reservada y ambigua ${ }^{5}$ de las Academias puede haberse visto influida por la importante monografía de Martín Camacho (2002), quien intentó desbaratar completamente la hipótesis sobre la interfijación en español y se dio el trabajo de analizar todos los ejemplos clásicos que aparecen reproducidos prácticamente en todos los tratados de la formación de palabras (p. ej. Lang 1992; Miranda 1994; Almela 1999) desde la publicación del primer artículo sobre el tema (Malkiel 1958).

\section{Perspectiva diacrónica}

Pasemos ya al primer problema que dificulta el análisis de las formaciones parasintéticas e interfijadas. La condición de que no debe existir una fase intermedia (una voz prefijada o sufijada), previa a la formación parasintética o interfijada, acusa innegablemente una perspectiva histórica e imposibilita la aplicación satisfactoria de criterios exclusivamente sincrónicos. Recordemos que el concepto de parasíntesis fue propuesto aún antes del nacimiento del estructuralismo, así que la condición de la simultaneidad de la adjunción de un prefijo y sufijo a la base fue establecida por Darmesteter todavía en el contexto de la lingüística diacrónica. Dentro del marco teórico actual, la parasíntesis probablemente ni siquiera se hubiera planteado, hecho que corrobora también el paulatino abandono de la costumbre de tratar la parasíntesis como un procedimiento lexicogenético independiente. El problema es que, para determinar si una palabra aparentemente parasintética fue formada a partir de una voz previamente sufijada/prefijada o por la adición simultánea de dos afijos derivativos, el método más simple sigue siendo averiguar el origen de la formación. Algunos morfólogos trataron de prescindir en sus investigaciones del enfoque histórico, pero los resultados obtenidos plantean dudas sobre la viabilidad del empleo de criterios puramente sincrónicos. Puesto que no disponemos del suficiente espacio para exponer todas las dificultades que abarca el análisis sincrónico de los parasintéticos, baste decir que algunos gramáticos generativos (p. ej. Corbin 1987 y 1980) incluso llegaron a negar el concepto de parasíntesis con dos argumentos interesantes: a) en la parasíntesis verbal, el sufijo (típicamente -ar: allanar, embotellar, etc.) no es un morfema derivativo, sino flexivo; y b) la fase intermedia no atestiguada no es relevante, ya que en la lengua en cuanto sistema, las palabras posibles (no existentes, pero perfectamente

\footnotetext{
5 En el capítulo sobre la derivación apreciativa, los académicos prefieren interpretar las formaciones del tipo buenecito como palabras sufijadas en vez de interfijadas, analizando el posible interfijo como parte del sufijo. No obstante, al referirse a palabras como Carlitos, Osquitar, lejitos o azuquítar -que algunos lingüistas clasifican como voces infijadas porque el afijo diminutivo aparece delante del segmento final de la palabra primitiva-, los autores de la NGLE (2009: 640-641) admiten que la opción de considerar -it- como interfijo es aquí más convincente que en otros casos. Para colmo, en los apartados dedicados a la derivación aumentativa (pág. 657) se reconoce la posibilidad de incluir el supuesto interfijo -arr- de la palabra nubarrón en la base (nubarr-ón), con lo que nos encontramos, en las páginas de una sola gramática, con tres opciones totalmente distintas.
} 
imaginables a partir de las formaciones análogas) deberían tener el mismo estatuto que las palabras existentes, lo que pone en duda el criterio de simultaneidad de la doble afijación postulado desde el comienzo para la delimitación de los parasintéticos.

Ahora bien, ¿cuáles son las consecuencias concretas de la aplicación de los criterios sincrónicos para el análisis estructural de las formaciones supuestamente parasintéticas?

En primer lugar, y desde el punto de vista formal, varias voces construidas ya en latín podrían considerarse parasintéticas (p. ej. el verbo abreviar).

En segundo lugar, para etiquetar una palabra como parasintética, es necesario que el hablante común sea capaz de reconocer o analizar la base léxica (hablamos, pues, del criterio de transparencia), algo que no ocurre por ejemplo en el caso de los verbos agazapar(se) y acelerar (a pesar de existir el nombre gazapo y el adjetivo célere), como se menciona en la NGLE (2009: 582). El enfoque sincrónico no presenta muchas dificultades cuando se analiza la estructura de palabras como embotellar, enzarzar o alunizar (Pref+B+Suf), pero según advierte Serrano-Dolader (1995: 31), hay algunas formaciones -entre ellas el verbo desnivelar- donde se ofrece más de un análisis posible. A primera vista, esta palabra no parece satisfacer la condición básica establecida en la definición tradicional del proceso de parasíntesis, pues en español está atestiguado tanto el sustantivo desnivel como el verbo nivelar. Aun así, la descomposición morfológica basada en el criterio semántico demuestra que sí sería posible incluir el verbo desnivelar entre los parasintéticos. Según el lingüista zaragozano, es válido el análisis de esta voz como una formación prefijada (des+nivelar) con la acepción de «acción opuesta a nivelar», pero se puede proponer, para esta misma palabra, igualmente una estructura parasintética (des+nivel+ar) con el significado de «hacer perder el nivel». Cabe preguntarse, en tal caso, si estamos ante un verbo polisémico o si se trata de dos voces homónimas. Otro problema ligado a un análisis puramente sincrónico de dicha palabra plantea la imposibilidad de determinar, sin recurrir a consideraciones de carácter cronológico, si desnivelar con el primer significado mencionado («acción opuesta a nivelar») fue creado a partir del verbo nivelar o bien partiendo del sustantivo ya prefijado desnivel.

Semejantes dificultades surgen también en el estudio de las palabras interfijadas, lo que puede ilustrarse con un ejemplo citado en la NGLE (2009: 511): cafetero. Como afirma Portolés (1999: 5053), acérrimo defensor del concepto de interfijo en español y autor del capítulo respectivo en la GDLE (1999), «no son interfijos los segmentos que, formando parte de extranjerismos o de voces latinas, no sirvan o hayan servido para la creación léxica en nuestra lengua. Por esta razón, no son voces interfijadas los galicismos cafetera o tutear (del francés cafetière, tutoyer) o catalanismos como ferretero (del catalán ferreter)». Según el mismo lingüista, tampoco contienen interfijos los sufijos verbales -ear (<-idiare), -ecer (<-escere), -ificar (<-ificare) e -izar (<-izare), aunque sí son voces interfijadas verbos como al-et-ear, llor-iqu-ear o pint-arr-ear/pint-arr-aj-ar (donde tenemos incluso una cadena de interfijos). Es obvio que para analizar correctamente los verbos terminados en -ear, -ecer, -ificar e -izar, hay que contar con ciertos conocimientos de la morfología histórica del español, ya que un análisis puramente mecánico podría conducir a la conclusión errónea de que p. ej. el verbo languidecer contiene el mismo interfijo -ec- que entra en la formación del diminutivo pececito. No es necesario enumerar aquí todas las características que diferencian una secuencia formada por un interfijo y un sufijo de un simple sufijo o sus alomorfos, pero la condición básica para poder analizar una formación como interfijada es que esta no debe ser una palabra sufijada a la que se adjuntó posteriormen- 
Algunos problemas del análisis de formaciones interfijadas y parasintéticas en español

te otro sufijo. Portolés (1999: 5043) pone como ejemplo la voz ganadería, cuya creación se realizó en dos pasos sucesivos: ganado>ganadero>ganadería. Lógicamente, la única manera como averiguarlo es buscar si existe en español la fase intermedia; no obstante, ello ya significa, al igual que en el análisis de los parasintéticos, recurrir a un criterio diacrónico.

\section{Aspecto semántico}

Examinemos ahora el aspecto semántico de la interfijación y la parasíntesis. El incierto o nulo significado del interfijo es sin duda una de las razones más convincentes para no otorgar a los elementos interfijados el estatuto de afijo. Ya el autor del primer artículo sobre la interfijación en español (Malkiel 1958: 107) definió el interfijo como un elemento «falto de significado propio», y también Portolés (1993: 340) trató de justificar su estatuto derivativo citando la muy famosa y demasiado amplia definición de afijo de Aronoff (1981: 16, apud Portolés 1993: 340): «Lo esencial del morfema: no es lo que signifique sino simplemente que seamos capaces de reconocerlo». En la GDLE (1999), el propio Portolés abandona este postulado y pretende demostrar que al menos una parte de los interfijos aportan cierto significado a la base (p. ej. el sema de «abundancia» en hum-ar-eda, polv-ar-eda, viv-ar-acho, etc. $\left.{ }^{6}\right)$. Donde sí puede apreciarse un valor semántico incontestable es en las palabras que contienen un interfijo que coincide en su forma (y parcialmente en su significado) con un sufijo apreciativo (cant-urr-ear, pint-orr-ear, llor-iqu-ear), pero precisamente estos «morfemas» pueden ser interpretados como sufijos encadenados, así que el inventario de los verdaderos interfijos quedaría reducido a elementos completamente desprovistos de significado, como el segmento -c- en mujer-c-ita o padre-c-ito. Varela (2005: 35-36) considera los interfijos «morfos vacíos» por no cumplir con una de las tres condiciones fundamentales para asignar a una secuencia de fonemas el estatuto de morfema, es decir, «que sea una unidad significativa mínima». Almela (1999: 183) admite que «no es fácil reconocer en el interfijo el significado», pero argumenta que la aportación sémica del interfijo puede averiguarse al menos de manera negativa, es decir, mediante su eliminación. Según este autor, se aprecia una diferencia de significado en las palabras con o sin interfijo como lavotear/lavar; quejicoso/quejoso, apretujar/ apretar; sin embargo, todos los interfijos implicados funcionan en español también como sufijos diminutivos o aumentativos/despectivos, de modo que no se trata exactamente de ejemplos representativos. En cualquier caso, otros interfijos no poseen ningún matiz significativo y su función es meramente morfofonemática (cursilería, camioncito, invernadero), y lo mismo puede afirmarse del elemento de enlace - $i$ - (en los compuestos del tipo pelirrojo), que lingüistas como Dressler (1986) o Almela (1999) también incluyen entre los interfijos. Portolés (1993: 347) apunta, además, que el interfijo no modifica directamente el significado de la base, sino el del sufijo siguiente, lo cual explicaría las dificultades en la determinación del valor exacto de estos elementos.

También la parasíntesis presenta no pocos problemas a la hora de asignar un significado concreto a los afijos implicados en este proceso. Si nos limitamos a la parasíntesis verbal, parece claro que el prefijo en- contenido en embotellar («meter el vino u otro líquido o producto en botellas») o encarcelar («meter a alguien en la cárcel») ${ }^{7}$ tiene valor locativo, pero incluso aquí puede haber algunas dudas. Como advierte Serrano-Dolader (1995: 113), no es posible identificar el prefijo con la preposición utilizada en la paráfra-

\footnotetext{
Véase Portolés (1999: 5059).

Véase DRAE (2001).
} 
sis del parasintético, como muestra el ejemplo siguiente: aprisionar: «meter a alguien en prisión» o «llevar a alguien a prisión ${ }^{8}$. A diferencia de la prefijación estándar, donde el valor semántico del morfema guarda una relación más o menos estrecha con la preposición correspondiente (al menos en lo que a los prefijos con correlato preposicional se refiere), «el significado de la formación parasintética integra combinadamente los de prefijo, base y sufijo, sin que parezca posible matizar separadamente el valor específico del prefijo en cada caso» (Serrano-Dolader 1999: 4703). Recordemos que semejante argumento esgrime Portolés (1993) cuando admite dificultades para atribuir un significado específico al interfijo.

Aunque no se trata de casos muy frecuentes, en español también pueden hallarse formaciones con o sin prefijo que tienen significado idéntico: baldosar-embaldosar, balsamarembalsamar. Además, el mismo valor causativo se expresa, incluso con bases antonímicas, mediante la adición del prefijo o prescindiendo de él (sucio>ensuciar, limpio>limpiar; véase Serrano-Dolader 1995: 105), y esta misma alternancia se observa igualmente en verbos casi sinonímicos (embellecer/hermosear), lo que dificulta la asignación de una función concreta al prefijo.

\section{Carácter flexivo o derivativo del sufijo verbal}

La última cuestión que quisiéramos abordar en este breve repaso de los problemas teóricos relacionados con la parasíntesis y la interfijación concierne al estatuto derivativo o flexivo del afijo verbal. Pongamos por caso los verbos ensuciar, atontar y apretujar. El sufijo -ar podría interpretarse aquí como un morfema flexivo (desinencia) y no derivativo, con el consiguiente análisis de estas formaciones como verbos prefijados o sufijados, respectivamente. Es bien sabido que los límites entre la derivación y la flexión son borrosos, pero en la actualidad la mayoría de los lingüistas se inclinan por reconocer a la terminación del infinitivo la función derivativa en determinadas palabras. Eso parece relativamente obvio cuando se forman p. ej. verbos denominales o deadjetivales (limpio>limpiar); no obstante, también hay verbos interfijados con bases verbales (apretar>apretujar) en los que el sufijo no produce ningún cambio categorial, así que el segmento -ar podría analizarse simplemente como una desinencia y, por consiguiente, apretujar no sería una palabra interfijada sino sufijada. La argumentación de Portolés (1999: 5046-5047) en contra de este razonamiento es como sigue: los mismos interfijos que aparecen delante del sufijo verbal se utilizan igualmente para la formación de nombres (peg-uj-ón $n_{\mathrm{N}} \mathrm{x}$ apret-uj-ar $\mathrm{V}_{\mathrm{V}^{\prime}}$ grand-ull-ón $_{\mathrm{N} / \mathrm{A}} \times$ masc-ull-ar ${ }_{\mathrm{V}}$ ) en los que no puede haber ninguna duda acerca del carácter sufijal del segmento final, de modo que «defender la existencia de interfijos tanto en nombres como en verbos se acomoda mejor a la morfología del español que limitar la interfijación a un proceso de la morfología nominal» (pág. 5047).

Un problema similar se presenta en la parasíntesis verbal, que es el modelo parasintético más productivo en español. Si reconocemos al prefijo la capacidad transcategorizadora (y parece que en las palabras del tipo antiarrugas el prefijo de hecho desempeña esta función), sería concebible pensar que también en verbos como agrandar o abaratar, el verdadero responsable del cambio categorial pudiera ser el prefijo (o bien el circunfijo formado por un prefijo transcategorizador y el sufijo verbal). El mayor argumento a favor de este análisis se apoya en que la forma del infinitivo es solo una convención lexicográ-

\footnotetext{
8 Serrano-Dolader menciona además la existencia del verbo alternativo emprisionar, que no está recogido en el DRAE (2001).
} 
fica y que en algunas formas personales o finitas no queda ni un rastro del supuesto «sufijo verbalizador»: agrand-ar>agrand-o. En otras formas (agrand-a-s, agrand-a-mos) aparece al menos la vocal temática, hecho que llevó a algunos morfólogos a atribuir el estatuto de afijo derivativo (verbal) solo a esta, pero en cualquier caso, la primera persona del singular del presente del indicativo no conserva ni siquiera la vocal temática, así que el problema teórico en cuestión no puede resolverse satisfactoriamente de esta manera. Serrano-Dolader (1995: 56-60) trató de salvar dicho obstáculo con el recurso al concepto de morfema cero (en nuestro caso la vocal temática); la NGLE (2009: 579) no rechaza explícitamente esta explicación y se limita a hacer constar, como en muchos otros casos, que «no hay acuerdo general sobre cuál sea la mejor solución de este problema teórico». A pesar de la vacilación mencionada, los académicos prefieren considerar, para los fines de la descripción de los esquemas de derivación y parasíntesis verbal, todo el segmento final (-ar, -er, -ir) como sufijo derivativo.

\section{Conclusiones}

Para terminar, cabe resumir los tres aspectos comentados en los apartados 3 a 5 .

Tanto el concepto de parasíntesis como el de interfijación dependen inseparablemente del orden cronológico de la adjunción de los morfemas derivativos, por lo que no siempre resulta posible diferenciar nítidamente los casos de derivación secundaria de los procesos de parasíntesis o interfijación, respectivamente. Las dificultades teóricas arriba comentadas dimanan también de la evolución de la lingüística en general y de la morfología en particular: como ya hemos dicho, la definición de la parasíntesis se remonta a la lingüística preestructural, así que la condición de la simultaneidad de la adición de un prefijo y un sufijo a la base es prácticamente una reliquia del pasado. Lo mismo puede aducirse, incluso en doble sentido, del concepto de interfijación. Si dejamos aparte el criterio de inexistencia de la fase intermedia, la posibilidad de etiquetar como afijo un segmento sin ningún valor semántico o función gramatical (mujer-c-ita) se debió a una concepción demasiado amplia del morfema (formulada por el generativista Aronoff) con la que Malkiel y Portolés lograron justificar la inclusión de elementos muy heterogéneos en el mismo grupo de los llamados interfijos. El paulatino abandono de la propuesta de Aronoff forzó a Portolés a modificar su teoría, pero el problema de la ausencia de cualquier rasgo semántico en muchos interfijos quedó irremediablemente enquistado en su concepción hasta hoy día.

El problema de la aportación semántica del afijo concierne también a la parasíntesis. Mientras que en la prefijación la productividad neológica del morfema antepuesto está condicionada por la conciencia lingüística de los hablantes sobre su significado, en los esquemas parasintéticos más productivos ( $a$-/en-+B+-ar/-ecer) la asignación de un valor concreto al morfema inicial se complica simplemente por el hecho de que el significado del parasintético no resulta solo de la fusión de los semas de la base y el prefijo, sino también de su combinación con el sufijo verbal. Esta circunstancia hablaría a favor de la hipótesis de que el hablante, a la hora de crear un nuevo parasintético, no es consciente del significado del prefijo y sufijo tomados aisladamente, sino que trabaja con esquemas que incluyen ambos afijos a la vez y que conducen a una modificación semántica previsible (p. ej. la causatividad). Desde esta perspectiva, la idea del circunfijo no parece tan descabellada y probablemente acierta a describir mejor el proceso neológico implicado. 
En cuanto al tercer y último punto mencionado, hay que constatar que el debate sobre el estatuto derivativo o flexivo del sufijo verbal tiene relevancia solo para el análisis de los verbos con interfijo (p. ej. apretar>apret-uj-ar) ${ }^{9}$, los cuales, por otro lado, representan buena parte del inventario de las voces interfijadas. Sin querer negar la validez de la interpretación alternativa, creemos que la necesidad de cierta consistencia en el tratamiento de las formaciones derivadas aconseja atenerse a la postura tradicional y considerar el sufijo verbal como morfema derivativo, o bien prescindir completamente del concepto de interfijación y analizar los interfijos en español como sufijos o meros elementos de enlace, según proponen varios lingüistas. Con ello quedaría resuelto no solo el problema del carácter flexivo o derivativo del morfema verbal en las formaciones interfijadas, sino también las demás dificultades arriba mencionadas.

\section{Bibliografía}

Almela Pérez, Ramón (1999), Procedimientos de formación de palabras en español, Barcelona: Ariel.

Aronoff, Mark (1981), Word Formation in Generative Grammar, Cambridge: MIT.

Bosque, Ignacio - Demonte, Violeta (dirs.) (1999), Gramática descriptiva de la lengua española, Tomo III, Madrid: Espasa-Calpe [GDLE en el texto].

CoRBIN, Danielle (1987), Morphologie dérivationnelle et structuration du lexique, Tübingen: Max Niemeyer.

CoRbin, Danielle (1980), «Contradictions et inadéquations de l'analyse parasynthétique en morphologie dérivationnelle», in: Dessaux-BerTHONNEAU, AnneMarie (ed.), Théories linguistiques et traditions grammaticales, Lille: Presses universitaires de Lille, 181-224.

Darmesteter, Arsène (1875), Traité de la formation des mots composés dans la langue française comparée aux autres langues romanes et au latin, Paris: Librairie A. Franck.

DRESSLER, Wolfgang Ulrich (1986), «Forma y función de los interfijos», Revista Española de Lingüística 16 (2), 381-395.

FERNÁNDEZ AlCALDE, Héctor (2010), «La estructura léxico-sintáctica de los verbos parasintéticos», Interlingüística 20 [Disponible en: http://filcat.uab.cat/clt/XXIVAJL/ Interlinguistica/Encuentro\%20XXIV/Fernandez_Alcalde_REVF.pdf].

LANG, Mervyn Francis (1992), Formación de palabras en español, Madrid: Cátedra.

Malkiel, Yakov (1958), «Los interfijos hispánicos. Problema de lingüística histórica y estructural», in: Miscelánea Homenaje a André Martinet, Tomo II, La Laguna: Universidad de La Laguna, 107-199.

Martín Camacho, José Carlos (2002), El problema lingüístico de los interfijos españoles, Cáceres: Universidad de Extremadura.

Miranda, José Alberto (1994), La formación de palabras en español, Salamanca: Ediciones Colegio de España.

Portolés, José (1999), «La interfijación», in: Boseue, Ignacio - Demonte, Violeta (dirs.), Gramática descriptiva de la lengua española, Tomo III, Madrid: EspasaCalpe, 5041-5073.

Portolés, José (1993), «Sobre los interfijos en español», in: VARela OrTEGa, Soledad (ed.), La formación de palabras, Madrid: Taurus, 339-359.

\footnotetext{
9 La parasíntesis verbal supone un procedimiento heterogéneo (luna>alunizar, gordo>engordar, etc.), por lo que es más fácil defender el carácter derivativo del morfema final.
} 
Real Academia Española (2001), Diccionario de la lengua española, Madrid: EspasaCalpe [DRAE en el texto].

Real Academia Española - Asociación de Academias de la lengua Española (2009), Nueva gramática de la lengua española, Madrid: Espasa Libros [NGLE en el texto].

SERRANO-DOLADER, David (1999), «La derivacion verbal y la parasíntesis», in: BosQuE, Ignacio - Demonte, Violeta (dirs.), Gramática descriptiva de la lengua española, Tomo III, Madrid: Espasa-Calpe, 4683-4755.

SERrano-Dolader, David (1995), Las formaciones parasintéticas en español, Madrid: Arco/Libros.

Štichauer, Pavel (2007), Tvoření slov v současné italštině, Praha: Nakladatelství Karolinum.

Varela Ortega, Soledad (2005), Morfología léxica: La formación de palabras, Madrid: Gredos.

\author{
Petr Stehlík \\ Ústav románských jazyků a literatur \\ Filozofická fakulta \\ Masarykova univerzita v Brně \\ Arna Nováka 1 \\ 60200 Brno \\ República Checa \\ stehlik@phil.muni.cz
}

